\title{
Linx
}

Revue des linguistes de l'université Paris X Nanterre

5 | 1994

La négation

\section{L'insoutenable légèreté morphologique du préfixe négatif in- dans la formation d'adjectifs}

J.C. Anscombre

\section{(2) OpenEdition}

\section{Journals}

Édition électronique

URL : http://journals.openedition.org/linx/1225

DOI : $10.4000 /$ linx.1225

ISSN : 2118-9692

Éditeur

Presses universitaires de Paris Nanterre

\section{Édition imprimée}

Date de publication : 1 juin 1994

Pagination : 299-321

ISSN : 0246-8743

\section{Référence électronique}

J.C. Anscombre, «L'insoutenable légèreté morphologique du préfixe négatif in- dans la formation d'adjectifs », Linx [En ligne], 5 | 1994, mis en ligne le 18 juillet 2012, consulté le 06 mai 2019. URL: http://journals.openedition.org/linx/1225 ; DOI : 10.4000/linx.1225

Ce document a été généré automatiquement le 6 mai 2019.

Département de Sciences du langage, Université Paris Ouest 


\title{
L'insoutenable légèreté morphologique du préfixe négatif in - dans la formation d'adjectifs
}

\author{
J.C. Anscombre
}

\section{Introduction}

1 Cette étude est le premier volet d'une étude plus vaste consacrée à l'étude des préfixes dits négatifs. Nous nous bornerons ici à évoquer le cas de in- dans le cas de la formation d'adjectifs. Cette dénomination de "préfixe négatif» vient de ce que dans un nombre appréciable de cas, il existe une paire de type (adjectif en in-, adjectif) telle que : être adjectif en in- $=$ ne pas être adjectif. Ainsi : (attendu, inattendu), (habité, inhabité), (possible, impossible), (stable, instable), (traduisible, intraduisible), et bien d'autres encore. Position classique fort clairement exprimée dans Togeby ; 1985 : « ... Les deux préfixes négatifs in- et anti- s'ajoutent uniquement à des noms (substantifs ou adjectifs)... in- se joint surtout à des adjectifs. Adjectifs dérivés en -able et -ible: incroyable, intraduisible; participes passés: inattendu, insoupçonné; adjectifs verbaux en -ant: impuissant, incroyant, insouciant ; autres adjectifs dérivés : impersonnel, infructueux. Il existe aussi des adjectifs simples qui admettent le préfixe in-: indigne, inégal, injuste, impur, etc., mais les adjectifs les plus fréquents, qui forment des couples d'antonymes, ne l'admettent jamais : bon-mauvais, grand-petit, haut-bas, large-étroit, long-court, vraifaux, beau-laid... » (p. 15).Cette position fait immédiatement surgir un certains nombre d'interrogations. On peut par exemple se demander pourquoi les sujets parlants identifient sans hésiter le in- de impotent comme ce même préfixe négatif, alors que l'on n'a pas potent. Invoquer l'emprunt (effectif) au latin n'explique rien. Pourquoi ne pas avoir emprunté les deux termes (comme dans le cas de docile/indocile), puisque potens existe en latin, et que par ailleurs, potent apparaît dans certains composés (omnipotent, idempotent, nihilpotent, plénipotentiaire)? 
2 Pour tenter d'y voir plus clair, nous commencerons par relever un certain nombre de phénomènes. A la lumière de ce qu'ils font apparaître, nous proposerons une solution reposant sur une perspective sémantique du problème, ainsi que les critères qui la complètent. Dans un dernier temps enfin, nous évoquerons notre conception des relations entre sémantique et morphologie.

\section{Faits et problèmes}

3 Si l'on établit une liste suffisamment longue d'adjectifs positifs et/ou négatifs, on remarque alors que :

a) Sans même aborder le problème de la définition exacte de «adjectif simple » et de "couples d'antonymes", il reste à expliquer pourquoi certains adjectifs simples admettent le préfixe négatif in- (ainsi apte/inapte) et d'autres non (par exemple profane) *im-profane). Par ailleurs, la notion d'antonyme n'explique rien. Car il existe des couples d'antonymes dont l'un des termes admet cette préfixation. Ainsi : exact/erroné, inexact ; clément/sévère, inclément; fidèle/volage, infidèle; modeste/prétentieux, actif/passif, inactif; docile/rétif, indocile, etc.

b) Que c'est parmi les adjectifs en -able/-ible et les adjectifs participes (présents et passés) que l'on trouve le plus de préfixation en -in.

c) Que lorsque la préfixation en in- est possible, elle introduit parfois, de façon apparemment imprévisible, des distorsions sémantiques importantes. C'est le cas des paires pitoyable/impitoyable, narrable/inénarrable, payable/impayable, désirable/indésirable, pensable/impen-sable, calculable/incalculable, etc.

d) Dans un certain nombre de cas, la préfixation en in- est strictement impossible : considérable/*inconsidérable, souhaitable/*insouhai-table, méprisable/ *imméprisable, taché/*intaché, permissif/*impermissif, étanche/*inétanche, préoccupé/ *impréoccupé, etc.

e) On pourrait penser à expliquer le point précédent d) en assortissant la dérivation morphologique de contraintes sémantiques. On se heurte alors au fait qu'il existe des adjectifs négatifs en in- sans contrepartie positive: *convenant/inconvenant, *peccable/ impeccable, *péni-tent/impénitent, *solite/insolite, *colore/incolore (mais on a coloré), *odore) inodore (mais on a odorant).

f) Enfin, pour exprimer des propriétés très proches, il y a des doubles couples (parfois même formés sur une même racine) au comportement étrange : (audible, inaudible)/(*ouï, inoui), (construisable, inconstruisable)/(constructible, *inconstructible), (habituel, inhabituel)/ (*solite, insolite), (buvable, imbuvable)/(potable, *impotable), (soucieux, insoucieux)/ (*souciant, insouciant), etc.

4 On peut d'ores et déjà remarquer que ces quelques faits sont totalement contraires à l'idée d'un préfixe négatif in- que l'on concatènerait à un mot (un adjectif, en l'occurrence), même agrémentée de contraintes sémantiques et de la notion « d'emprunt au latin $»^{1}$.

5 En fait, on se rend compte dès ce stade de l'importance de l'aspect sémantique dans cette dérivation ${ }^{2}$. Ainsi, inlouable signifie toujours 'qui ne peut être mis en location', et jamais 'qui ne peut faire l'objet de louanges'. Touchable a pour sens 'avec qui on peut entrer en contact', et non 'à qui on peut porter atteinte', alors que intouchable est ambigu de ce point de vue. Alors que désirer a deux sens, 'éprouver du désir' et 'vouloir', désirable ne 
renvoie guère qu'au premier, et indésirable uniquement au second. On peut multiplier les exemples de ce type.

6 Nous voudrions compléter ces remarques en y incluant deux critères syntaxiques proposés par Gaatone ; 1987, qui montre que la dérivation en in- fait perdre à la base ses propriétés verbales :

Ce livre est parfaitement lisible (pour + par) tout le monde.

Ce livre est parfaitement illisible (pour + * par) tout le monde.

Un règlement clair et applicable aussi.

Un règlement pas clair et non applicable non plus.

*Un règlement pas clair et inapplicable non plus

(Gaatone, 1987).

7 Critères qui montrent que l'explication de certains doublets comme une même base verbale mais une opposition formation populaire/formation savante, pose des problèmes, comme on le voit sur :

Cette eau est buvable par tout le monde.

${ }^{*}$ Cette eau est potable par tout le monde.

8 Nous voudrions ici proposer une approche qui fait de la sémantique le point de départ de la dérivation en in-. Comme les adjectifs représentent des propriétés, nous commencerons par nous pencher sur la notion même de propriété.

\section{Des différents types de propriétés}

9 Nous commencerons par donner un certain nombre de définitions, puis nous les illustrerons par des exemples montrant à quels types de comportement linguistique on peut les rattacher.

\section{Définition 1}

Nous dirons d'une propriété $P$ qu'elle est une propriété intrinsèque d'une entité I si P est constitutive de I. Une propriété qui n'est pas intrinsèque sera dite extrinsèque.

10 Ainsi, le couple malade/maladif fait jouer l'opposition extrinsèque/ intrinsèque. On est maladif par nature, et malade par accident. Aux yeux de la langue, les propriétés intrinsèques sont permanentes, alors que les propriétés extrinsèques sont - ou peuvent être présentées comme le résultat d'un procès. D'où des différences de comportement comme :

Pierre est tombé (malade + *maladif).

Pierre est gravement (malade + *maladif).

Chaque fois qu'il voit un serpent, Pierre est

(malade + *maladif) de peur.

J'ai vu Pierre hier, il était (malade + *maladif).

Définition 2

Une propriété $\mathrm{P}$ est essentielle pour une entité I si elle est définitoire de la classe des I. Dans le cas contraire, la propriété sera dite accidentelle si elle définit seulement une sous-classe d'entités I.

11 Par exemple, avoir deux bras est une propriété essentielle pour 'homme', alors que 'blond' ou 'brun' n'est qu'une propriété accidentelle. Par ailleurs, tant les propriétés essentielles que les accidentelles sont intrinsèques. Ce qui n'empêche pas des exemples comme :

Hier, Marie était blonde, aujourd'hui, elle est brune. 
Mais il ne s'agit plus des propriétés intrinsèques accidentelles 'blonde' et 'brune', sinon de propriétés extrinsèques résultant de la réalisation d'un procès (se teindre, en l'occurrence).

La langue distingue les propriétés essentielles et accidentelles par différents procédés. en particulier (Anscombre; 1990), dans les constructions de type $N_{1}$ à $N_{2}, N_{2}$ est nécessairement une propriété intrinsèque accidentelle de $N_{1}$. Cette propriété ne sera donc jamais essentielle, d'où des exemples comme :

J'ai vu un (fox à poil ras + canard à trois pattes).

J'ai vu un (*fox à poil + *canard à deux pattes).

Du même tonneau :

J'ai vu (*un homme à barbe + une femme à barbe).

J'ai acheté un vélo (*à pédales + à selle de course).

Par ailleurs, l'opposition entre $N_{1}$ à $N_{2}$, et $N_{2}$ avec $N_{2}$ est de type intrinsèque/extrinsèque.

D'où la différence entre jouet à piles et jouet avec piles :

Tous nos jouets sont livrés ( ? ? à + avec) piles.

Tous nos jouets fonctionnent (à + ? ?avec) piles.

Tous nos jouets fonctionnant à piles sont livrés avec piles.

On explique de façon analogue avion $(* \grave{a}+$ avec) réservoir supplémentaire : avoir un réservoir supplémentaire ne définit pas un type d'avion, et ne saurait donc constituer (en général) une propriété intrinsèque acciden-telle, mais seulement une propriété extrinsèque, d'où la préposition avec. On aura en revanche avion à géométrie variable.

Dernière remarque : les adjectifs désignant des propriétés intrinsèques accidentelles sont classifiants au sens de Milner; 1978. On les reconnaît au fait qu'ils admettent la plupart du temps la substantivation avec article défini: les rouges, les bruns, les hypocrites, les humains, etc. Ce test n'est pas absolu (il est suffisant, mais non nécessaire) : ? ?les maladifs ,? ?les potables, etc. Enfin, il y a aussi des classifiants parmi les adjectifs dénotant des propriétés extrinsèques : les blessés, les punis, etc.

On voit donc quel va être notre propos : relier les phénomènes signalés plus haut à propos de la "préfixation» en in- à cette classification des propriétés que nous venons d'esquisser sommairement, et qui est résumée par le graphe ci-dessous :

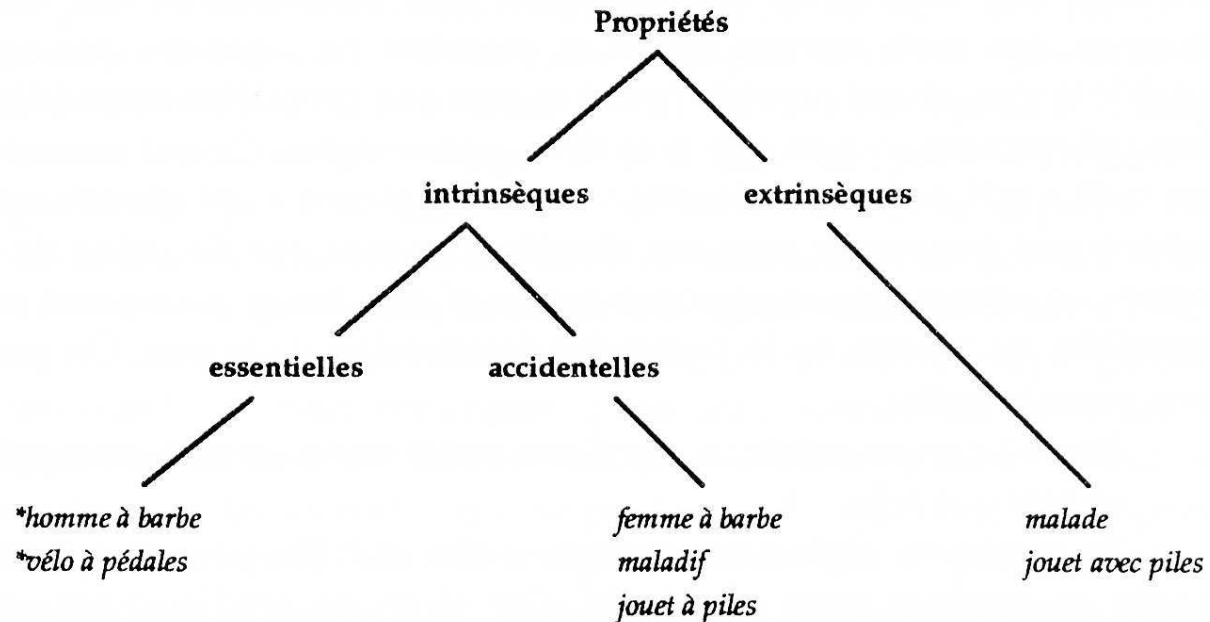




\subsection{Les propriétés essentielles (intrinsèques)} sous-classe. C'est également le cas de la prédication définitoire : les propriétés essentielles de la classe que l'on définit sont accidentelles pour les classes englobantes envisagées. On peut donc dire dans les deux cas que les propriétés prédiquées sont en fait accidentelles. Dans le cas de la déviation, c'est évident. Dans le cas de la définition, les propriétés sont accidentelles pour des classes plus grandes, et la définition sert précisément à dire que leur conjonction constitue une sous-classe pour laquelle elles sont essentielles. C'est pourquoi des mots comme voyant et entendant ne signifient pas 'qui possède la vue' et 'qui possède l'ouïe' en tant que propriétés essentielles, mais uniquement en tant que propriétés accidentelles. Ce qui revient à dire qu'il s'agit de termes classifiants qui s'opposent à aveugle et sourd. Remarquons que voyant peut être classifiant en tant que déviation de la norme : un voyant est une personne qui « voit » des choses qui ne sont pas accessibles au travers de la possession (essentielle) de la vue. On peut donc poser la règle :

Règle 1 : on ne prédique pas d'une entité une propriété essentielle en tant que telle.

En quoi cette règle nous concerne-t-elle ici ? Elle peut en fait être utilisée en quelque sorte au négatif. Car si on ne prédique pas une propriété essentielle d'une entité qui la possède comme telle, rien n'empêche de dire qu'il y a des exceptions, que certaines entités sont extraordinaires quant à cette propriété. D'où imberbe, qui signifie non pas 'sans barbe', mais 'sans barbe parce qu'il ne peut en avoir' (cf. un adolescent imberbe). Il est donc normal que l'on n'ait pas berbe. L'homme est berbe par essence, par nature. Et si l'on veut évoquer cette pilosité, on le fera par le biais d'une propriété accidentelle comme barbu. Remarquons au passage que ces adjectifs en - $u$ ne servent pas à présenter une propriété essentielle comme telle, mais à indiquer une réalisation hors-norme de cette propriété. Ainsi : chevelu, poilu, ventru, joufflu, dentu, fessu,... etc. On comprend de cette façon l'existence de inodore et incolore face à *odore et ${ }^{*}$ colore. Par essence, tout a une odeur et une couleur. Mais on peut exprimer que l'odeur et la couleur sont exceptionnelles en utilisant odorant et coloré. Ce qui nous permet d'éclaircir un petit mystère, celui du couple insipide/sapide. On remarque immédiatement que la relation n'est pas d'antonymie. Peu utilisé, sapide relève en fait du langage technique de l'art culinaire et de la chimie (les corps sapides). Il s'agit donc d'un classifiant renvoyant à une classification de la sapidité, et 
l'on comprend pourquoi. Puisqu'il ne peut renvoyer à une propriété essentielle en vertu de la règle 1 , sapide ne peut désigner qu'une propriété accidentelle. Ou bien il est classifiant - solution choisie par la langue, ou bien il renvoie à une déviation. Ce second rôle a été dévolu à savoureux, qui est en fait le véritable antonyme de insipide. Autre couple: *muable/immuable. Le fait que l'on n'ait pas l'adjectif traduit le simple fait que rien ne dure et que tout change : tout est par essence "muable». Les exceptions font donc partie de ce qui est immuable. On opposera ce couple à un autre couple à la fois proche et différent, à savoir changé/inchangé. Différence qui montre qu'une propriété résultant d'un procès ne peut être essentielle. On remarquera d'ailleurs que ce type de «trou lexical» se rencontre fréquemment dans le cas de doubles couples. Ainsi (*potent, impotent), (puissant, impuissant). Evoquer un emprunt (effectif) au latin n'explique rien: tant potens que impotens existent, et on ne voit guère pourquoi on n'aurait pas emprunté les deux termes, comme dans le cas de possible/impossible. Puisque impotent = 'qui est dans l'incapacité de se mouvoir', potent - s'il existait - signifierait «qui a la capacité de se mouvoir'. Cette capacité étant inhérente à tout être vivant ne peut être prédiquée, d'où * potent. On ne le trouvera donc qu'en composition, où il signale alors un écart d'avec la norme : omnipotent, plénipotentiaire ${ }^{3}$. Pour ce qui est du couple (puissant, impuissant), on peut remarquer qu'ils sont formés sur un écart par rapport au sémantisme de base 'qui peut'. En effet, puissant = 'qui peut beaucoup' et impuissant = 'qui ne peut rien'. Il s'agit donc de propriétés de type accidentel, l'analyse complète n'ayant pas sa place ici. Notons que les deux termes ne sont pas à proprement parler antonymes, comme il apparait sur des oppositions comme :

Cet animal a une musculature (puissante $+*$ impuis-sante).

Max est (*puissant + impuissant) à réagir ${ }^{4}$.

\subsection{Les propriétés intrinsèques accidentelles}

Etant donné qu'il y a des propriétés extrinsèques qui sont ou paraissent classifiantes, elles ne sont pas toujours faciles à identifier. Le point délicat est de déterminer si la propriété étudiée est intrinsèque ou extrinsèque on a vu de ses caractéristiques linguistiques. D'une façon très générale, les propriétés extrinsèques résultent d'un procès agentif, alors que ce n'est pas le cas (linguistiquement parlant) pour les intrin-sèques. Ce qui signifie que les adjectifs dénotant le résultat d'un procès agentif seront, moyennant certaines restrictions qui seront évoquées plus loin, à classer dans les désignateurs de propriétés extrinsèques. Nous verrons plus loin à propos de l'opposition décidé/résolu que si l'on parvient à séparer les intrinsèques des extrinsèques, ce n'est pas là chose facile, et encore moins évidente. Nous commencerons par étudier quelques cas d'adjectifs dénotant visiblement des propriétés intrinsèques accidentelles. Pour ce qui est de la préfixation en in-, on constate le comportement illustré par les exemples ci-après :

Marie est spécialisée dans les métaux (ferreux + précieux + non-ferreux + nonprécieux $+*$ inferreux $+*$ imprécieux).

Nous vivons dans une société (permissive + non-permissive $+*$ impermissive).

Le boîtier de ma montre est (étanche + non-étanche + *inétanche).

L'eau de ce puits est (potable + non-potable $+*$ impotable).

Ce terrain est (constructible + non-constructible $+*$ inconstructible).

Cette marque fabrique exclusivement des manteaux (réversibles + non-réversibles +

*irréversibles). 

précieux forment des sous-classes stables de la classe plus vaste des métaux, de la même façon que les montres étanches sont un type de montre bien précis. Une société permissive est un type de société qui s'oppose à une autre type, à savoir une société répressive. Constructible relève de la classification des terrains, et s'oppose à agricole et forestier (termes qui signifient tous deux non-constructible dans cette classification). De même, réversible est classifiant dans le domaine vestimentaire; dans le même ordre d'idée, signalons en peau (retournée + *irretournée). L'exemple de l'eau potable est intéressant dans la mesure où (potable, *impotable) s'oppose à (buvable, imbuvable). On a de façon tout à fait parallèle (comestible, *incomestible)/(mangeable, immangeable). Notre explication sera que potable est une propriété intrinsèque accidentelle (et donc classifiant au sens de Milner), alors que buvable est une propriété extrinsèque (qui est attribuée à la suite d'une évaluation externe). On comparera de ce point de vue :

Il y a tellement de chlore dans l'eau du robinet qu'elle est de moins en moins (*potable + buvable).

L'eau (non-potable + ? ?non-buvable $+*$ imbuvable) peut contenir des microbes dangereux. différence entre les deux adjectifs. Eau est massique, eau potable est également massique, mais eau buvable ne l'est pas:

Ce puits fournit une eau (buvable + imbuvable + potable + non-potable).

Ce puits fournit de l'eau ( ?buvable + ? ?imbuvable + potable + non-potable). ainsi bon-mauvais, grand-petit, haut-bas, vrai-faux, etc. - n'admettent jamais (ou presque) la dérivation en in-. Ils dénotent tous des propriétés intrinsèques accidentelles, et sont d'ailleurs classifiants au sens de Milner. On en déduit que dans les cas digne-indigne, purimpur, juste-injuste, etc., on a affaire à des propriétés extrinsèques: en l'occurrence, la propriété est attribuée au vu d'une norme externe - par exemple un jugement. Si d'ailleurs on utilise l'un des termes en un sens classifiant, la dérivation en in- ne donne pas un adjectif classifiant. D'où des contrastes comme :

Ce catalyseur est fait de métal (pur + ? ?impur + mélangé à des impuretés).

Autre conséquence : si pour un couple d'antonymes supposés, la dérivation est possible, c'est qu'il ne s'agit pas véritablement d'anto-nymes, et que la dérivation introduit une distorsion sémantique. Considérons par exemple le couple (intelligent, bête). De façon au premier abord surprenante, on a bel et bien inintelligent. Par ailleurs, on a également le couple (hypocrite, *inhypocrite). En fait, intelligent est susceptible d'être classifiant ou qualifiant. En tant que classifiant, son antonyme « naturel » est non-intelligent :

Si l'on en croit les résultats de ce test, Pierre ferait partie des intelligents, et Max des non-intelligents.

Le sens qualifiant supporte la combinaison avec Je trouve ...:

Je trouve ce type (intelligent + ? ?non-intelligent + inintelligent). 

classifiants :

Il évitait tout contact avec ses collègues, surtout avec les hypocrites.

Je trouve ce type (intelligent + ?hypocrite). terme marqué, et qu'un terme marqué ne prend apparemment jamais le préfixe in- ${ }^{5}$. Par ailleurs, dans la mesure où il s'interjecte (Ce type, quel hypocrite!) et ne s'interroge guère (Est-ce que Max est (franc + ? ?hypocrite)), il semble difficile de lui refuser des usages Autre opposition plutôt curieuse, le cas de (capable, incapable) et (compétent, incompétent). Tout le problème est que tant capable que incompétent ont des allures de classifiants. En fait, les apparences sont une fois de plus trompeuses. Il y a en effet deux constructions :

Pour ce poste, Marie a posé sa candidature : elle est (capable + *incapable).

Dans cet emploi, capable est classifiant et dénote la possession d'une certaine capacité, d'où *incapable. Seconde construction :

Max est (capable + incapable) de s'en tirer tout seul.

Il s'agit cette fois de l'évaluation d'une capacité à réaliser une certaine action, i. e. d'une propriété extrinsèque, et les deux adjectifs existent. Remarquons que l'on peu $t$ distinguer les deux emplois. Par exemple :

?? J'estime Max capable.

J'estime Max (capable + incapable) de s'en tirer tout seul.

Pour ce qui est de (compétent, incompétent), il s'agit cette fois d'une évaluation de la conformité à une norme : un tribunal compétent, un chirurgien incompétent, etc. Là encore donc, les deux adjectifs désignent des propriétés extrinsèques. Notons la différence de construction entre capable et compétent :

Pour ce poste, Marie a posé sa candidature : elle est (capable + compétente + incompétente).

Pour ce qui est de l'informatique, Marie est (*capable + compétente + incompétente) en la matière.

Dernier exemple : celui de l'adjectif périssable. Tout en ce bas monde devant périr un jour ou l'autre, on peut penser se trouver en présence d'un adjectif dénotant une propriété essentielle, ce qui serait un contre-exemple à $\mathrm{R}_{1}$. On remarque fort heureusement que le substantif le plus souvent présent en combinaison est denrée. Nous sommes ainsi renvoyé au champ sémantique du commerce, et périssable y est classifiant: on a des denrées périssables de la même façon que l'on a des denrées alimentaires. Il s'agit d'une nomenclature. Impérissable n'est pas l'antonyme de périssable, en conformité avec $\mathrm{R}_{2}$ - il n'y a pas de denrées impérissables. En revanche, on a souvenir impérissable ( = 'impossible à oublier'), mais non souvenir périssable : le souvenir est par nature éphémère.

\section{Agentivité et dérivation des adjectifs en in-}

37 On aura remarqué que dans les exemples précédemment étudiés, les adjectifs dénotant des propriétés extrinsèques semblaient nettement moins problématiques que ceux renvoyant à des propriétés intrinsèques. Très grossièrement, dans le cas des propriétés extrinsèques - et hors certaines configurations que nous caractériserons plus loin, tant l'adjectif "positif» que son "antonyme » en in- existent. Par ailleurs, nous avons à plusieurs reprises mentionné le fait que les propriétés extrinsèques semblent résulter 
d'un procès. D'où l'idée que la formation des adjectifs en in- pourrait bien être liée à l'existence d'un tel procès. Nous irons en fait plus loin, et tenterons de montrer le bienfondé de la règle :

$\mathbf{R}_{\mathbf{3}}$ : l'existence d'adjectifs en in- est liée à l'existence d'un procès agentif en relation

avec la propriété dénotée par l'adjectif considéré.

Notons qu'il n'est pas nécessaire - même si c'est là un cas fréquent - que le verbe dénotant ce procès soit morphologiquement apparenté à l'adjectif étudié. Nous avons vu que ce procès peut être un jugement d'attribution.

Nous voudrions présenter quelques arguments en faveur de cette hypothèse.

\subsection{L'argument de trous lexicaux}

Un premier argument nous sera fourni par l'existence de «trous lexicaux». Nous avions par exemple vu que l'on a constructible, mais *inconstructible. En revanche, on a simultanément construisable et inconstruisable. Nous avons vu que constructible désignait une propriété intrinsèque accidentelle, et ne supportait pas la dérivation en in- à ce titre. On peut donc s'étonner qu'il n'en soit pas de même pour construisable. Il est en fait facile de voir que la différence entre les deux adjectifs est la processivité de construisable face à la stativité de constructible :

? ? Ce terrain est facilement constructible.

? ? Ce terrain est constructible en un mois.

*Ce terrain est constructible par n'importe quel maçon.

Cette maison est facilement construisable.

Cette maison est construisable en un mois.

Cette maison est construisable par n'importe quel maçon.

Il peut d'ailleurs se faire qu'un même adjectif admette ou non la contre-partie en in- selon que l'interprétation en est processive ou non. Par exemple :

Ce manteau est (réversible + *irréversible).

Cette réaction chimique est (réversible + irréversible).

De façon intuitive, un manteau réversible est un type de manteau, la réversibilité faisant partie de sa nature même. Une réaction chimique en revanche suppose, pour être renversée, une intervention externe. Il s'agit toujours d'une propriété, mais cette fois extrinsèque. ce que l'on peut voir sur des contrastes comme :

??Ce manteau est difficilement réversible.

Cette réaction chimique est difficilement réversible.

A haute ou à basse température, cette réaction chimique est réversible.

*Qu'il fasse beau ou qu'il fasse mauvais, ce manteau est réversible.

On a un cas très semblable avec perméable/imperméable :

Cette firme est spécialisée dans les vêtements (*perméables + imperméables).

Les terrains calcaires sont généralement (perméables + imperméables).

Dans le cas du vêtement, on peut comprendre qu'un imperméable soit un type de vêtement, au contraire de "un perméable $»^{6}$. Aucune industrie textile n'a pour but la confection de tels vêtements. Mais on reste alors perplexe devant le second cas : pourquoi un terrain par exemple perméable ne serait-il pas un certain type de terrain? Pour expliquer ce phénomène, nous partirons de l'étude de Milner ; 1982, qui fait remarquer que des noms comme description, construction, communication,... etc., ont la plupart du temps deux interprétations, l'une stative, l'autre processive, avec des propriétés 
syntactico-sémantiques différentes. Il nous semble qu'il en est de même pour certains adjectifs, dont perméable. Dans un premier sens, statif, perméable signifie quelque chose comme 'non-étanche'. En ce sens, aucun vêtement n'est imperméable : nos vêtements sont conçus pour nous protéger des intempéries, non pour nous en isoler totalement. Dans un second sens, perméable donne lieu cette fois à une lecture processive, à savoir 'que les liquides traversent'. L'interprétation stative fait de perméable une propriété intrinsèque essentielle. Par nature, un vêtement est perméable tout comme un homme a de la barbe. Il peut se trouver en revanche que par extraordinaire, un vêtement soit imperméable de la même façon qu'un homme peut être imberbe. La lecture processive à l'inverse, fait de perméable une propriété extrinsèque : perméable = 'que l'eau (ou les liquides) traversent', qui s'oppose à perméable = 'qui a la capacité à être traversé'. On remarquera que ces deux lectures se rencontrent également dans l'opposition sauveteur = 'qui a la capacité à sauver' et sauveur = "qui sauve ". Il nous reste donc à montrer que dans terrain perméable, il s'agit bel et bien de l'interprétation processive. Or l'interprétation processive correspond à la construction perméable à/imperméable à. En effet, avec cette construction, on a :

Ce maillot de bain est (perméable + imperméable) aux U.V.

Dans cet exemple, il ne peut s'agir de la propriété intrinsèque, puisqu'alors les deux adjectifs ne sont pas possibles simultanément. Il ne peut donc s'agir que d'une propriété extrinsèque, le à servant à introduire l'agent du procès. Or dans notre exemple des terrains, il s'agit de la perméabilité non pas aux liquides (ou à tout autre chose) en général, mais aux eaux de pluie ou de ruissellement. Cet exemple est donc à lire comme :

Les terrains calcaires sont généralement (perméables + imperméables) aux eaux de pluies.

i. e. donne lieu à une interprétation processive, laquelle autorise l'adjectif et son antonyme en in-.

Un dernier exemple, celui de l'opposition décidé/résolu, va illustrer le rôle crucial de l'interprétation processive dans la formation des adjectifs en in-. Les deux adjectifs semblent en effet très proches par le sens, ainsi dans :

La garnison était (décidée + résolue) à lutter jusqu'au bout.

Par ailleurs, on remarque l'opposition *indécidé/irrésolu, qui montre que les deux adjectifs étudiés n'appartiennent pas à la même catégorie sémantique. Nous voudrions développer ici quelques arguments en faveur d'une lecture stative de décidé et d'une lecture processive de résolu. Une première différence apparaît au niveau des verbes morphologiquement apparentés se décider et se résoudre:

Pierre est en train de (se décider + * se résoudre) à intervenir dans la discussion.

Pierre s'est facilement ( ? ?décidé + résolu) à tout abandonner.

Selon Zribi-Hertz; 1987, de tels contrastes distinguent les interprétations réfléchies agentives (se résoudre) et les réfléchies ergatives (se décider). Ce contraste se retrouve au niveau des adjectifs. Résolu, au contraire de décidé, caractérise uniquement des états passagers, jamais des traits permanents :

Max a (un air décidé + une démarche décidée + un caractère décidé).

Max a un (air résolu + une démarche résolue + ? ?un caractère résolu).

Max est une personne (décidée + ? ?résolue).

Enfin, ce qui n'est pas le cas de décidé, résolu signifie toujours résolu à :

Pour ce qui est de l'offre de travail qu'on lui a faite, Marie est (décidée + résolue) à se battre. 
Pour ce qui est de l'offre de travail qu'on lui a faite, Marie est (décidée + ? ?résolue).

$51 \mathrm{Au}$ vu de ces arguments, décidé est donc lu comme statif, résolu comme processif. De façon quelque peu lapidaire, décidé = 'qui sait prendre une décision' et résolu = 'qui a pris une décision'. Nos règles nous font alors prévoir irrésolu et *indécidé. Pour exprimer le concept correspondant à ce dernier, on doit passer par indécis, avec un décis qui n'existe pas (mais on a existé en ancien français au sens de 'décidé'). On peut comprendre ce qui s'est passé : résolu désignant la propriété extrinsèque, et décidé la propriété intrinsèque accidentelle, décis en est venu à dénoter la propriété intrinsèque essentielle. Il a donc disparu comme tel, mais non indécis, en vertu de nos règles.

\subsection{Les adjectifs dérivés de verbes météorologiques}

Une caractéristique importante des verbes météorologiques, mise en évidence dans Ruwet; 1990, entre autres articles, est qu'ils sont non-agentifs, en fait ergatifs. On peut donc s'attendre raisonnablement à ce que les adjectifs décrivant des états météorologiques ne soient susceptibles que d'une interprétation stative (de type classifiant), et que par conséquent, l'antonyme en in- n'existe pas. Ce que l'on vérifie sur des exemples comme :

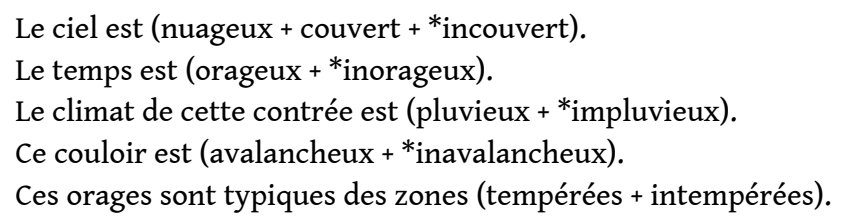

53 Notons que la façon la plus naturelle de former les antonymes correspondants est ici, une fois de plus, d'avoir recours à la préfixation en non-.

\subsection{Les adjectifs dénotant des états pathologiques}

Les états pathologiques (ainsi les maladies) sont subis par l'organisme attaqué. L'humain siège d'un état pathologique est donc pris dans un procès où il n'a pas le rôle d'agent. Les verbes à sujet humain et exprimant une relation entre ce sujet et une maladie (attraper, choper, couver, souffrir, être victime de, être sujet à...) sont notoirement non-agentifs. Pour ce qui est des adjectifs correspondants (lorsqu'ils existent), on remarquera leur combinaison avec des verbes soit ergatifs, soit d'état :

Pierre est tombé malade.

Le petit paraît fiévreux.

Marie est devenue alcoolique.

Max est resté fragile (des poumons).

On ne s'étonne donc pas de constater que la préfixation en in- là encore n'est pas possible :

Pierre est (malade $+*$ immalade).

Le petit est (fiévreux + *infiévreux).

On a fait le test sur des populations (tuberculeuses + non-tuberculeuses +

*intuberculeuses).

Marie est (blessée + *imblessée).

En élargissant un peu le champ des états pathologiques, on notera le contraste :

Pierre est (guéri + non-guéri + *inguéri) de ses blessures de guerre (interprétation stative). 
Les blessures de guerre de Pierre sont parfaitement (guérissables + inguérissables)

(interprétation processive).

\subsection{Adjectifs désignant des états psychologiques}

57 Plusieurs études menées sur les verbes psychologiques ont montré que l'endroit où se manifeste et se développe un sentiment n'est ni un agent, ni un patient (i. e. il n'a les propriétés linguistiques ni de l'un, ni de l'autre), mais un 'lieu psychologique'. Ce qui se reflète, au niveau des noms apparentés, par des propriétés particulières (cf. Anscombre, 1992, 1993) qui les apparentent aux noms d'état. Et qui se répercute bien entendu au niveau adjectival. Les adjectifs dont tout porte à croire qu'ils dénotent des états psychologiques n'admettent pas l'antonyme étudié :

Max est (répugnant + *irrépugnant).

Marie est (horrifiée $+*$ inhorrifiée).

Pierre est (réservé + *irréservé).

Marie est (consternée + *inconsternée). éprouve du respect', soit d'une interprétation processive 'qui montre du respect', et irrespectueux ne peut, selon nos règles, être issu que de la seconde. Nous rejoignons là une analyse de Kupferman; 1991, qui remarque cette même double possibilité pour le substantif respect. C'est selon nous l'origine de l'opposition :

Pierre éprouve (du respect + * de l'irrespect) pour ses supérieurs.

Pierre montre (du respect + de l'irrespect) envers ses supérieurs.

61 Pour ce qui est des adjectifs, on peut faire ressortir la différence entre les deux lectures à l'aide d'adverbes qui forcent la lecture processive :

Pierre est (hypocritement + facilement + ouver-tement) respectueux (??des

sentiments des autres + envers les supérieurs).

Marie est une femme (désirable + *indésirable).

C'est là un sort (désirable + indésirable). 
Cet individu est (*désirable + indésirable) sur le sol national. et l'autre processive, issue de désirer = 'requérir, demander'. Conformément à ce que nous
avons dit, l'antonyme n'existe que pour la seconde lecture. Lecture pour laquelle c'est
désirable qui n'est pas possible. Nous verrons plus loin pourquoi.

\subsection{Quelques cas épineux}

Nous voudrions montrer ici que les hypothèses que nous avons exposées plus haut permettent de résoudre au moins partiellement certain cas particulièrement ardus. Nous commencerons par la double paire habituel/inhabituel, *solite/insolite. Vu la proximité sémantique, il est loisible de se demander le pourquoi de *solite. Invoquer l'emprunt au latin n'explique rien:: le latin classique possédait solitus/insolitus, tous deux dérivés du semi-déponent soleo 'avoir coutume de'. Habituel provient en revanche d'un emprunt au latin médiéval, qui nous renvoie cependant au déponent habituor du latin classique, 'avoir coutume de'. Les dérivés nominaux vont toutefois nous fournir un précieux indice. En effet, habitus signifie 'maintien, manière d'être', alors que solitum est fréquemment traduit par '(chose) ordinaire' (magis solito = 'plus que d'ordinaire'). On voit quelle est notre hypothèse : soleo signifierait 'être habitué par nature (selon l'ordre des choses)', alors que habituor serait 'être habitué à faire'. Dans la terminologie de Zribi-Hertz ; 1987, soleo serait un ergatif, et habituor un intransitif. Cet écart sémantique se serait conservé dans les substantifs dérivés, solitum dénotant une propriété intrinsèque essentielle, et habitus une propriété extrinsèque. Cette distinction se serait conservée en latin médiéval, se serait transmise aux adjectifs solitus/habitualis, puis aux adjectifs français correspondants. Ce qui expliquerait *solite, mais habituel/inhabituel. Bien entendu, il faudrait une étude philologique détaillée pour confirmer ces hypothèses. On peut cependant remarquer qu'il subsiste certains indices en français contemporain. Ainsi Le meuble était à une place inhabituelle signifie que le meuble ne se trouvait pas où il était habituellement. En revanche, Le meuble était à une place insolite veut dire que le meuble occupait une place qui n'est pas celle d'un meuble.

Dernier exemple, celui du triple couple *mense/immense, mesurable/*immesurable, commensurable/incommensurable. De immense = 'au-delà de toute mesure', on déduit que * mense signifierait quelque chose comme 'qui a une mesure'. Or selon cette signification, le monde perceptible par les humains est mense. Il n'y a d'ailleurs aucune langue qui exprime le dépassement de toute mesure par un mot simple, i. e. non dérivé. Il s'agit toujours d'un composé signifiant 'non-fini, non-calculable'. *Mense exprimerait ainsi une propriété intrinsèque essentielle, et n'existe pas en vertu de $R_{1}$. On a en revanche immense, représentant la déviation d'avec cette norme. Et comme cela est fréquent (cf. infini, incalculable, inaccessible), prend fréquemment un sens extrême. Pour ce qui est de commensurable, on remarque qu'il n'entre que dans des constructions comme :

$\mathrm{X}$ est commensurable avec $\mathrm{Y}$.

$\mathrm{X}$ et $\mathrm{Y}$ sont commensurables entre elles.

et que l'on n'a jamais :

$\mathrm{X}$ est commensurable.

67 Ce qui suggère que commensurable suppose une comparaison, i. e. un procès dont la commensurabilité serait le résultat. Dans ces conditions, il s'agirait d'une propriété extrinsèque, donnant lieu de façon régulière à incommensurable : 
La diagonale du carré est incommensurable avec son côté.

empêche d'y voir l'expression d'une propriété intrinsèque essentielle. Il ne peut donc
s'agir que d'une propriété essentielle accidentelle, et comme telle soumise à $\mathrm{R}_{2}$. En fait, le
verbe-base mesurer possède deux emplois bien distincts, à savoir:

Cette pièce mesure vingt mètres carrés.

Pierre a mesuré cette pièce.

71 (Willems, 1981). En fait l'existence de la construction réflexive Une telle pièce se mesure montre que le second type d'emploi se subdivise en un emploi transitif à interprétation processive, et un emploi réflexif à se-moyen, à interprétation stative (cf. Zribi-Hertz, 1982). Nous ferons l'hypothèse que mesurable correspond à cette interprétation stative, d'où *immesurable. On peut se demander si mesurable est susceptible d'une lecture processive.

Il semble qu'il en soit ainsi dans un exemple comme :

Ces radiations sont mesurables par les méthodes habituelles.

Il est remarquable qu'existe alors un antonyme en in-, caractérisé par la violation de la règle phonologique in $+m . . . \rightarrow$ imm... :

Ces radiations sont in-mesurables par les méthodes habituelles.

74 Nous ne voudrions pas clore ce paragraphe sans signaler un exemple qui nous a été proposé par J. Anis (Paris X - Nanterre), celui de vertébré/invertébré. Selon les critères habituels, les vertébrés forment une classe stable, et une sous-classe du monde animal. D'après $\mathrm{R}_{2}$, invertébré ne devrait donc pas exister. En fait, les vertébrés ne sont pas ceux 'qui possèdent des vertèbres', mais qui exhibent toute une organisation, faisant intervenir, outre la colonne verticale et le squelette, les appareils respiratoires, nerveux et circulatoires. D'où vertébré = 'qui possède l'organisation V'. Il y a plus : cette définition est diachronique, les vertébrés étant le dernier stade d'une évolution qui part des invertébrés. Il faut donc lire vertébré comme signifiant 'qui possède l'organisation $\mathrm{V}$ au terme d'une certaine évolution'. Il s'agit là d'une lecture processive, d'où invertébré. En sciences naturelles d'ailleurs, invertébré ne s'oppose pas à vertébré, mais à (vertébré + protocordé), les protocordés (ou provertébrés) étant une classe voisine des vertébrés, mais moins évoluée.

\section{Formation des adjectifs en in- et stéréotypicité}

75 Nous voudrions maintenant esquisser le cadre théorique que nous envisageons pour traiter ce problème de la formation des adjectifs en in-, et plus généralement, les problèmes de formation morphologique où il semble que la sémantique joue un rôle fondamental.

76

En schématisant quelque peu, on peut dire que la dérivation en in- est généralement considérée comme étant le processus schématisé comme suit : 


\begin{tabular}{|c|c|c|}
\hline Adjectif ADJ & $\rightarrow$ dérivation & $\rightarrow$ Adjectif In-ADJ \\
\hline & $\uparrow$ & \\
& $\begin{array}{l}\text { Contraintes } \\
\text { sémantiques }\end{array}$ & \\
\hline
\end{tabular}

77 Cette position, qui fait de habité/inhabité la dérivation type des adjectifs en in-, échoue, nous l'avons vu, à expliquer bon nombre de phénomènes, par exemple ceux signalés à propos de puissant/impuissant. C'est donc une autre voie que nous adopterons. Nous commencerons par un certain nombre de définitions.

Définition 3 : étant donné une unité lexicale $\mathrm{M}$ (par exemple un adjectif), nous appellerons stéréotype une suite ouverte d'énoncés associée de façon permanente à $\mathrm{M}^{7}$.

Cette idée de stéréotype, que l'on trouvera développée dans Fradin ; 1984, Anscombre, 1990, est destinée à expliquer les contraintes syntaxiques et sémantiques qui régissent l'emploi des mots de façon parfois mystérieuse. Notons que le stéréotype ne se superpose pas à la signification, il est la signification.

Enfin, on remarquera la parenté entre la notion de stéréotype et d'autres notions que nous avons étudiées avec 0 . Ducrot, comme la polyphonie, les topoï et la délocutivité. Ainsi, Fradin ; 1984 montre que dans stylo, il y a un stéréotype comme/x écrire avec la plume/, et Anscombre; 1990, explique les propriétés de robe à fleurs à partir d'un stéréotype/Il y a des fleurs sur x/. Il y a ainsi différentes sortes de stéréotypes; en particulier, certains sont processifs, d'autres pas. L'idée que nous tentons de développer est la suivante : non seulement les stéréotypes sont attachés aux mots, mais ils peuvent être attachés à des radicaux. Notons au passage qu'un radical non verbal peut fort bien subsumer un stéréotype processif, ou l'inverse. Par ailleurs, deux radicaux ou même deux mots non morphologiquement apparentés sont susceptibles de mettre en jeu le même stéréotypes, ou des stéréotypes voisins. On peut ainsi imaginer que ouie, audition, oreille, entendre, proviennent d'un stéréotype commun. Possibilité que la langue exploite à fond par emprunt de radicaux latins, lui permettant ce faisant, d'opposer par exemple potable et buvable. La langue possède en outre un jeu de procédés dérivationnels $D_{1}, D_{2}, \ldots, D_{n}$, applicables aux mots ou aux radicaux, et qui possèdent trois caractéristiques principales : a) Ils déterminent la classe grammaticale du dérivé ; b) Ils considèrent, sémantiquement parlant, le stéréotype sous un certain point de vue. Schématiquement, sauveur voit le stéréotype/x sauve $\mathrm{y} / \mathrm{comme}$ un procès, sauveteur le voit comme une propriété (intrinsèque). Sauveur = 'celui qui sauve ou a sauvé', sauveteur = 'celui qui a la capacité à sauver'; c) Pour pouvoir être appliqués, ils exigent que soient remplies certaines contraintes sémantiques. Ainsi, le suffixe -eur qui sert à former des noms d'agent exigera l'agentivité du stéréotype d'où tueur mais *blesseur, *moureur. La philosophie de cette approche peut se résumer ainsi: les stéréotypes forment un système de concepts (sémantique). A l'aide des radicaux et des procédés dérivationnels en sa possession, la langue se 'débrouille' morphologiquement comme elle peut pour représenter ces concepts ou des concepts dérivés. Ce que l'on peut figurer sur le schéma ci-dessous : 


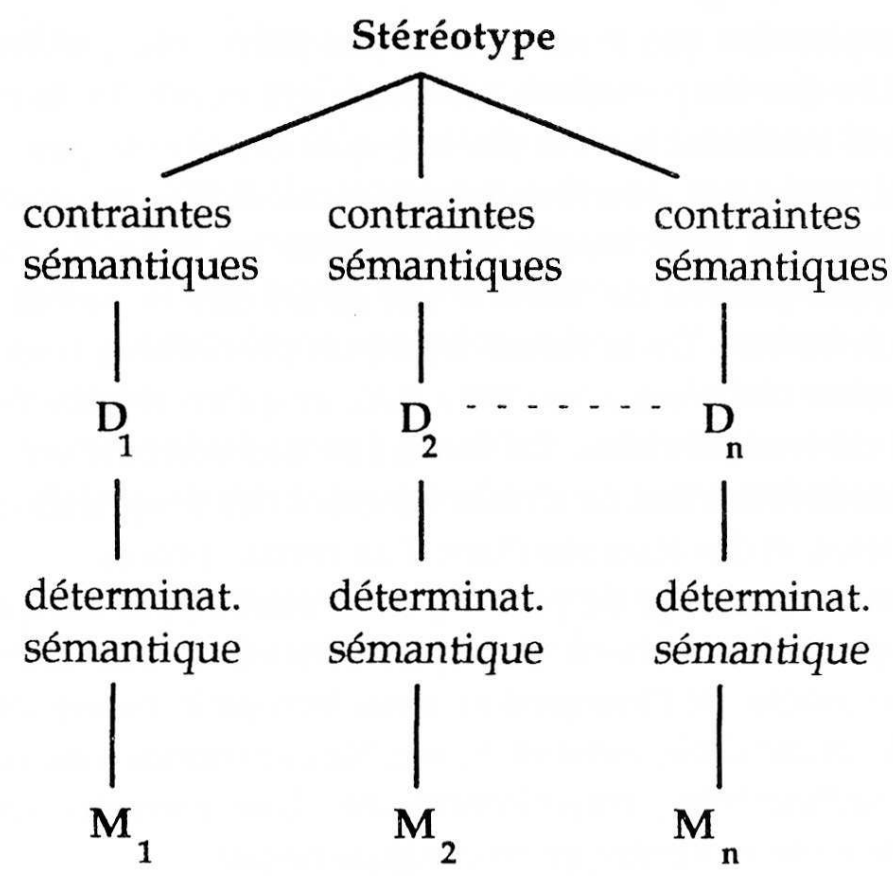

Quelle est la place des 'dérivés' adjectivaux en in- dans ce schéma? Nous avons vu que deux cas étaient possibles: ou bien l'adjectif dénotait une propriété intrinsèque essentielle, auquel cas il n'était pas dicible, mais l'adjectif en in- l'était, comme signalant une déviation d'avec la norme. Le second cas est celui où l'adjectif a une lecture processive. Il s'agit maintenant de regrouper les deux cas sous une même règle, autant que faire se peut. Nous ferons l'hypothèse suivante :

Règle de formation :

L'existence d'adjectifs en in- est reliée à l'existence d'une base processive. In- est la lexicalisation de la négation d'un stéréotype processif.

81 Nous avons dit que dans le cas des propriétés intrinsèques essentielles, l'adjectif dérivé indiquait un écart selon une norme naturelle. Notre règle de formation nous conduit à interpréter cette déviation comme le résultat d'un procès : si une entité ne possède pas la propriété intrinsèque essentielle Adj., c'est qu'elle a été le siège d'un procès dont l'état résultant est in-Adj. Qu'en est-il pour les propriétés extrinsèques? Raisonnons sur l'exemple :

Le phénomène $P$ était imprévisible.

Nous partirons du stéréotype grossier mais suffisant :

/X a prévu P/

qui donne par négation :

/X n'a pas prévu P/

Or cet énoncé ne signifie nullement que $\mathrm{X}$ n'a pas fait de prévisions. Il signifie simplement que $\mathrm{P}$ ne figurait pas parmi ces prévisions, ce qui revient à dire que ces prévisions comprenaient non-P. De la même façon, une quantité incalculable n'est pas une quantité qui ne peut faire l'objet d'un calcul, mais une quantité qu'aucun calcul n'aura comme résultat. On voit le rapport avec imberbe. Est imberbe un individu qui aurait dû être par nature pourvu de "barbité », et qu'un certain procès a mis dans l'état de non-barbité. De la même façon, un phénomène imprévisible est un phénomène qui a eu, a, ou aura lieu, et qu'un certain procès a mis dans l'état de non-prévision. La formation des adjectifs en in- 
apparait ainsi comme la formation de termes dénotant des propriétés qui sont des nonpropriétés, et des états résultant d'un certain procès.

Un autre avantage de passer par les stéréotypes est que l'on peut expliquer par cette méthode pourquoi certains termes ont une valeur sémantique proche de l'interjection, ainsi incroyable, impensable, invivable, inadmissible, incalculable, inénarrable, etc. Nous raisonnerons sur la double paire crédible/*incrédible, croyable/incroyable. Une première remarque est que croyable ne se rencontre qu'en contexte négatif :

Cette histoire (*est + n'est pas) croyable.

Cette histoire est (à peine + difficilement) croyable.

Crédible admet en revanche tant les contextes négatifs que les positifs. Mais il n'admet pas certains contextes négatifs que croyable affectionne particulièrement :

Cette histoire (est + n'est pas crédible).

Ce projet est ( ? ?à peine + ? ?difficilement) crédible.

Par ailleurs, le registre oral fait souvent usage de la combinaison pas croyable qui fonctionne comme un adjectif. Rien de tel dans le cas de crédible :

Max m'a raconté une histoire /pas croyable/.

Max m'a présenté un projet */pas crédible/.

Nous proposerons l'explication suivante. Crédible dénote en fait une propriété intrinsèque : est crédible une entité qui possède en soi les caractéristiques qui forcent la croyance. C'est pourquoi crédible supporte mal la combinaison avec un à peine fortement énonciatif ${ }^{8}$, ou avec un difficilement qui a une grande affinité avec les interprétations processives. Si on admet cette analyse, alors l'impossibilité de incrédible en découle. Pour ce qui est de incroyable, le fait que croyable n'existe qu'en contexte négatif ainsi que l'existence de ce curieux pseudo-adjectif /pas croyable/ nous indiquent la solution. Incroyable vient d'un stéréotype de la forme /Pas croyable, ce X/, /Je ne crois pas à ce $\mathrm{X} /$, /Ce $\mathrm{X}$, je n'en crois pas un mot/, où la négation est de type polémique au sens de Anscombre-Ducrot, performative au sens de Paricio, 1985. A l'aide de la notion de stéréotype, nous expliquons que certains adjectifs en in-, tels ceux cités plus haut, prennent d'emblée une valeur interjective: ils sont formés à partir de stéréotypes interjectifs. Nous pouvons même esquisser une explication du phénomène (*désirable + indésirable) sur le sol national. En effet, il y a une formule à valeur juridique « La présence de $\mathrm{X}$ n'est pas désirée sur le sol national, ayant force de loi, mais pour des raisons évidentes, il n'y a pas le correspondant «La présence de X est désirée sur le sol national ». Dernier point enfin: la notion de stéréotype telle que nous l'utilisons permet de comprendre le fréquent recours à des racines 'savantes'. Nous pensons que ce procédé sert en fait à indiquer soit que les dérivés ne le sont pas à partir du même stéréotype, soit qu'il s'agit du même stéréotype mais avec des spécifications (par exemple aspectuelles) différentes.

89 Bien entendu, un gros travail reste encore à faire au niveau de la détermination exacte des stéréotypes et de leurs spécifications. 


\section{BIBLIOGRAPHIE}

Anscombre (J.C.), 1990, " Pourquoi un moulin à vent n'est pas un ventilateur ", Langue française, $\mathrm{n}$ • 86, p. 103-25. 1992, «Quand on fait du sentiment : réflexions (presque) spontanées sur la nature linguistique des noms psychologiques ", in Hommages à Nicolas Ruwet, L. Tasmowski et A. Zribi-Hertz éds., Communication et cognition, Gand, p. 139-154. 1993, « Noms de sentiment, noms d'attitude, et noms abstraits ", à paraître dans Lexique.

Benveniste (E.), 1975, Noms d'agent et noms d'actions en indo-européen, Maisonneuve, Paris.

Corbin (D.), 1991, « La formation des mots : structures et interprétations », Lexique, n 10, p. 7-30.

Fradin (B.), 1984, « Anaphorisation et stéréotypes nominaux », Lingua, 64, p. 325-369. 1992, «Pourquoi la morphologie n'est pas configurationnelle », in Hommages à Nicolas Ruwet, L. Tasmowski et A. Zribi-Hertz éds., Communication et cognition, Gand, p. 229-243.

Gaatone (D.), 1987, « Les préfixes négatifs avec les adjectifs et les noms verbaux », Cahiers de lexicologie, $1, \mathrm{n}^{\circ}$ 50, p. 79-90.

Horn (L.R.), 1989, A Natural History of Negation, The University of Chicago Press, Chicago and London.

Kupferman (L.), 1991, « L'aspect du groupe nominal et l'extraction de en », Le Français moderne, 59, $\mathrm{n}^{\circ} 2$, p. 113-147.

Labelle (J.), 1986, « Grammaire des noms de maladie », Langue française, nº 69, p. 108-128.

Milner (J.C.), 1978, De la syntaxe à l'interprétation, Ed. du Seuil, Paris. 1982, Ordres et raisons de langue , Ed. du Seuil, Paris.

Muller (J.C), 1990, « Contraintes de perception sur la productivité de la préfixation verbale en dé négatif ", Travaux de linguistique et de philologie, XXVIII, p. 171-192. 1991, La négation en français, Droz, Genève.

Paricio (L. Hernández), 1985, Aspectos de la negación, Colección Contextos, Centro de estudios metodológicos e interdisciplinares, Universidad de León.

Pesetsky (D.), 1985, « Morphology and Logical Form », Linguistic Inquiry, 16, p. 193-246.

Picabia (L.), 1978, Les constructions adjectivales en français, Droz, Genève.

Ruwet (N.), 1990, « Des expressions météorologiques », Le Français moderne, 58, n 1/2, p. 43-97.

Togeby (K.), 1985, Grammaire française. vol. V : la structure de la proposition, Akademisk Forlag, Copenhague.

Willems (D.), 1981, Syntaxe, lexique et sémantique : les constructions verbales, Université de Gand, Gand.

Zribi-hertz (A.), 1982, « La construction se-moyen du français et son statut dans le triangle moyen-passif-réfléchi », Linguisticae Investigationes, VI, $\mathrm{n}^{\circ}$ 2, p. 345-401. 1987, « La réflexivité ergative en français moderne ", Le Français moderne, 55, n 1/2, p. 23-54. 


\section{NOTES}

1. Signalons au passage l'extrème ambiguïté de la notion d'emprunt à une langue étrangère. Nous pensons qu'il faut distinguer l'emprunt qui est intégré au système (par exemple redingote ou crédible), et le mot qui n'est utilisé que comme emprunt (ainsi ruling class).

2. «Dérivation » est pris ici, faute de mieux, dans un sens très général et flou.

3. Ainsi qu'en mathématiques nihilpotent, indempotent, équipotent.

4. Remarquons que la spécialisation au domaine sexuel ne change rien : puissant = «qui peut beaucoup sexuellement », et impuissant = « qui ne peut rien sexuellement parlant ».

5. L'étude détaillée reste à faire, d'autant plus que la notion de terme marqué est loin d'être claire.

6. Les vêtements sont en effet (selon la langue) conçus pour protéger du froid, ce que l'on peut voir sur un contraste comme :

Pierre s'est acheté des vêtements chauds pour l'hiver.

*Pierre s'est acheté des vêtements froids pour l'été.

Lorqu'ils ne sont pas spécialement conçus pour les intempéries, les vêtements sont donc par nature perméables.

7. Cette définition concerne uniquement le stéréotype primaire, attaché en permanence à $M$. Nous ne parlerons pas ici des stéréotypes secondaires qui peuvent être induits par le contexte.

8. On peut remarquer en effet que, comme les interjections, à peine ne s'interroge pas, et ne sert guère de réponse à une question. Par ailleurs, il est facile de constater qu'il n'aime guère les propriétés intrinsèques accidentelles: à peine ( ${ }^{*}$ maladif + ??content + ??potable), que l'on comparera à à peine (malade + buvable + lisible).

\section{RÉSUMÉS}

Le but de cet article est la prédictibilité de la formation d'adjectifs en français au moyen du préfixe négatifs in-. Un examen des différents problèmes montre que cette dérivation est largement sémantique, et dépend en particulier du type de propriété dénotée par la base. L'étude des différents types de propriétés permet d'établir les règles de dérivation, et montre l'importance du rôle joué par la processivité dans ce phénomène.

The aim of this article is to provide predictibility rules for word formation of French adjectives with regard to negative prefix in-. A careful examination of the problems involved shows that this word formation is mainly semantical, and crucially depends on the kind of property shown by the stem. A classification of the different kinds of properties leads to the corresponding attachment rules, and shows the importance of processivity in this phenomenon.

\section{AUTEUR}

\section{J.C. ANSCOMBRE}

L.A.P.S.A.R.L.A.C. 